\title{
The Lorentz Anomaly via Operator Product Expansion
}

\author{
Stefan Fredenhagen 1 , Jens Hoppeㄹ, Mariusz Hynek ${ }^{3}$ \\ ${ }^{1}$ Max-Planck-Institut für Gravitationsphysik, Albert-Einstein-Institut \\ Am Mühlenberg 1, 14476 Golm, Germany \\ ${ }^{2,3}$ Department of Mathematics, Royal Institute of Technology, KTH \\ 10044 Stockholm, Sweden
}

\begin{abstract}
The emergence of a critical dimension is one of the most striking features of string theory. One way to obtain it is by demanding closure of the Lorentz algebra in the light-cone gauge quantisation, as discovered for bosonic strings more than fourty years ago. We give a detailed derivation of this classical result based on the operator product expansion on the Lorentzian world-sheet.
\end{abstract}

\footnotetext{
${ }^{1}$ E-mail: stefan.fredenhagen@aei.mpg.de

2E-mail: hoppe@kth.se

${ }^{3} \mathrm{E}$-mail: mkhynek@kth.se
} 


\section{Contents}

1 Introduction

2 Operator product expansion and composite operators 4

3 Basic commutation relations $\quad 6$

4 The crucial commutator $\quad 8$

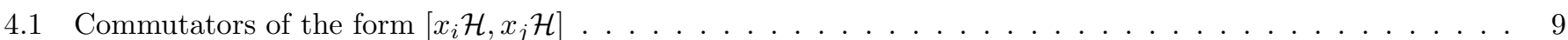

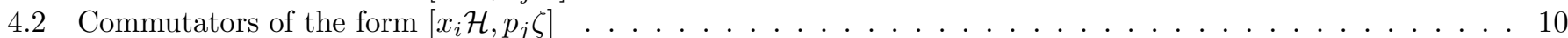

4.3 Remaining commutators and final result . . . . . . . . . . . . . . . . . . . . . . 12

A Commutators of the form $\left[p_{i} \zeta, x_{j} \mathcal{H}\right] \quad 12$

$\begin{array}{ll}\text { B Commutators of the form }\left[p_{i} \zeta, p_{j} \zeta\right] & 13\end{array}$

\begin{tabular}{lr} 
C Non-associativity & 15 \\
\hline
\end{tabular}

\section{Introduction}

More than forty years ago, 26 was noted as a critical dimension for the dual-resonance models that preceded string theory [1, 2, 3. One way of obtaining the critical dimension has been to show that in light-cone gauge quantisation the longitudinal Lorentz-operators $M_{i-}, i=1, \ldots, d>1$, only commute in $D=d+2=26$ space-time dimensions [4]. In this computation the generators $M_{i-}$ are normal-ordered infinite sums cubic in the oscillator modes of the quantised string 1 Whereas the quantisation of the string is well understood, much less is known for general $M$-dimensional extended objects; however, in [6] it was noted that, as a consequence of Lorentz invariance, a dynamical symmetry exists - which might give a way to algebraically determine the spectrum if one can understand this symmetry in the quantum theory. Classically, these higher-dimensional objects can be described - similarly to the string - in the light-cone gauge, but the corresponding world-volume theories are not free as in the case of strings. Therefore one does not have an expansion in terms of harmonic oscillators that would make it possible to quantise the theory directly. On the other hand, one can still use field theory techniques like operator-product expansions (OPE) in the computations.

These considerations motivated us to rederive the critical dimension of bosonic string theory in the light-cone gauge quantisation by only using the operator product expansion on the Lorentzian world-sheet. As the world-sheet theory is free, the OPEs are simple and completely equivalent to the harmonic oscillator commutators of the corresponding modes (so that it is guaranteed that the result is the same as in the oscillator approach). The computation, however, turned out to be surprisingly tedious and subtle (it involves a careful treatment of composite and non-local operators). We decided to write it up and present it in this note, in the hope that the approach might be useful for higher dimensional extended objects (note also [7]), - as well as an alternative derivation of the critical dimension (see also [8] where yet another derivation of the Lorentz anomaly was presented). We should add that, similar to the oscillator computation, it seems that one can not pinpoint any particular step of the computation where the anomaly arises; it rather appears as a result of an interplay of several anomalous terms that arise due to the regularisations needed to define the composite operators. Note however that, because we work on the (Lorentzian) cylinder, there is no need to artificially introduce a

${ }^{1}$ cp. [5]; standard textbooks have curiously refrained from presenting the calculation in detail 
normal-ordering constant in the computation; it is already set to the right value by using the most natural definition of composite operators.

Let us describe the computation in a language that can also be used for higher-dimensional extended objects (see 9] for some naive heuristic considerations). The string is parameterised by a map from the Lorentzian cylinder (time coordinate $t$ and angular variable $\varphi$ ) to a flat Minkowski space. In the light-cone gauge the degrees of freedom are carried by the transversal fields $\vec{x}$ and their conjugate momenta $\vec{p}$, as well as by the zero mode $\zeta_{0}$ of the coordinate $\zeta=x_{-}$and by its conjugate variable $\eta$.

Classically the longitudinal generators of the Lorentz algebra are given by

$$
M_{i-}:=\int_{0}^{2 \pi}\left(x_{i} \mathcal{H}-\zeta p_{i}\right) d \varphi, \quad i=1, \ldots, d=D-2 .
$$

Here

$$
\mathcal{H}(\varphi):=\frac{\pi}{\eta}\left(\vec{p} \cdot \vec{p}+\vec{x}^{\prime} \cdot \vec{x}^{\prime}\right)
$$

is the classical Hamiltonian density (corresponding to $p_{-}$), and

$$
\zeta(\varphi)=\zeta_{0}-\frac{2 \pi}{\eta} \int_{\varphi}^{2 \pi} \vec{p} \cdot \vec{x}^{\prime} d \psi+\frac{1}{\eta} \int_{0}^{2 \pi} \vec{p} \cdot \vec{x}^{\prime} \psi d \psi+\frac{\pi-\varphi}{\eta} \int_{0}^{2 \pi} \vec{p} \cdot \vec{x}^{\prime} d \psi
$$

is the reconstructed $x_{-}$coordinate of the string that follows from $\zeta^{\prime}=\frac{2 \pi}{\eta} \vec{p} \cdot \vec{x}^{\prime}$. The transversal fields $x_{i}(\varphi), p_{j}(\varphi)$ are constrained by

$$
\int_{0}^{2 \pi} \vec{p} \cdot \vec{x}^{\prime} d \varphi=0
$$

so that the last term in (1.3) could be dropped, and $\zeta$ can be rewritten as

$$
\zeta(\varphi)=\zeta_{0}+\frac{2 \pi}{\eta} \int_{0}^{\varphi} \vec{p} \cdot \vec{x}^{\prime} d \psi+\frac{1}{\eta} \int_{0}^{2 \pi} \vec{p} \cdot \vec{x}^{\prime} \psi d \psi .
$$

That the $M_{i-}$ Poisson-commute (provided (1.4) holds) is a particular case of a result of Goldstone [10, who for arbitrary dimension $M$ of the extended object solved

$$
\frac{\partial \zeta}{\partial \varphi^{a}}=\frac{\vec{p} \cdot \partial_{a} \vec{x}}{\eta \rho}, \quad a=1, \ldots, M
$$

for $\zeta$ in terms of $\vec{x}$ and $\vec{p}$ and some Green's function $G$ ( $\rho$ is a density satisfying $\left.\int \rho d^{M} \varphi=1\right)$ and then showed that classically, for all $M$, the generators of the inhomogeneous Lorentz group can be consistently realised on the $\left(\eta, \zeta_{0}, \vec{x}(\varphi), \vec{p}(\varphi)\right)$ phase-space constrained by the consistency of (1.6) (which for $M=1$ and $\rho=\frac{1}{2 \pi}$ simply becomes (1.4)). In the string case considered here,

$$
G(\varphi, \psi)=2 \pi(\psi-\varphi) \theta(\psi-\varphi)-\frac{1}{2}(\psi-\varphi+\pi)^{2}+\frac{\pi^{2}}{6},
$$

and the reconstructed $x_{-}$coordinate is

$$
\zeta(\varphi)=\zeta_{0}-\frac{1}{\eta} \int_{0}^{2 \pi} \partial_{\psi} G(\varphi, \psi) \vec{p}(\psi) \cdot \vec{x}^{\prime}(\psi) d \psi,
$$

which reduces to the expression (1.5) given above.

The paper is organised as follows. In section 2 we explain the OPE techniques that are needed to get to a quantum definition of the Lorentz operators. We then derive the commutation relations of all basic fields in section 3 , Finally, we compute the crucial commutator $\left[M_{i-}, M_{j-}\right]$ in section 4 . The three appendices contain some technical parts of the computation. 


\section{Operator product expansion and composite operators}

The definition of the Lorentz generators involves products of fields which we have to define properly in the quantum theory. In a free theory this can be done by using an oscillator expansion of the free fields and then define normal-ordered products by moving annihilation operators to the right of creation operators. Alternatively we can use the operator product expansion (OPE) of the fields to define composite operators by subtracting the singular part of the OPE. This leads to an equivalent description for free fields, but it can in principle also be used in more general situations where the usual normal ordering prescription in terms of annihilation and creation operators is not possible.

In the case at hand, the $x_{i}$ are massless free fields on the two-dimensional cylinder, and their OPE reads (no summation over $i$ )

$$
x_{i}(\tilde{\varphi}) x_{i}(\varphi)=-\frac{1}{2 \pi} \log \left|\sin \frac{\tilde{\varphi}-\varphi}{2}\right|+\text { regular } .
$$

Then the OPE of the fields $x_{i}^{\prime}$ is given by

$$
x_{i}^{\prime}(\tilde{\varphi}) x_{i}^{\prime}(\varphi)=S_{\text {sing }}(\tilde{\varphi}, \varphi)+\text { regular },
$$

with the distribution $S_{\text {sing }}$ in two variables $\tilde{\varphi}$ and $\varphi$ given by

$$
S_{\text {sing }}(\tilde{\varphi}, \varphi)=-\frac{1}{2 \pi} \partial_{\tilde{\varphi}} \partial_{\varphi} \log \left|\sin \frac{\tilde{\varphi}-\varphi}{2}\right|=\frac{1}{4 \pi} \partial_{\tilde{\varphi}}\left(\cos \left(\frac{\tilde{\varphi}-\varphi}{2}\right) \mathcal{P} \frac{1}{\sin \left(\frac{\tilde{\varphi}-\varphi}{2}\right)}\right),
$$

where $\mathcal{P}$ denotes the principal value. To define the operator " $x_{i}^{\prime}(\varphi) x_{i}^{\prime}(\varphi)$ " we use point-splitting, so we evaluate (2.2) for $\tilde{\varphi}=\varphi-\epsilon$ and determine the singular piece,

$$
\left.x_{i}^{\prime}(\tilde{\varphi}) x_{i}^{\prime}(\varphi)\right|_{\tilde{\varphi}=\varphi-\epsilon}=-\frac{1}{2 \pi \epsilon^{2}}+\text { regular } .
$$

Note that away from $\varphi=\tilde{\varphi}, S_{\operatorname{sing}}$ is a regular function, and we can replace $\tilde{\varphi}=\varphi-\epsilon$. This singular piece is then subtracted to define the product of $x_{i}^{\prime}$ with itself,

$$
\left(\left(x_{i}^{\prime} x_{i}^{\prime}\right)\right)(\varphi):=\lim _{\epsilon \rightarrow 0}\left(\left.x_{i}^{\prime}(\tilde{\varphi}) x_{i}^{\prime}(\varphi)\right|_{\tilde{\varphi}=\varphi-\epsilon}+\frac{1}{2 \pi \epsilon^{2}}\right) .
$$

Up to an additive constant this is equivalent to the normal ordering prescription using oscillators.

Similarly we have

$$
p_{i}(\tilde{\varphi}) p_{i}(\varphi)=S_{\text {sing }}(\tilde{\varphi}, \varphi)+\text { regular },
$$

and

$$
\left(\left(p_{i} p_{i}\right)\right)(\varphi):=\lim _{\epsilon \rightarrow 0}\left(\left.p_{i}(\tilde{\varphi}) p_{i}(\varphi)\right|_{\tilde{\varphi}=\varphi-\epsilon}+\frac{1}{2 \pi \epsilon^{2}}\right) .
$$

This then leads to the quantum definition of $\mathcal{H}$,

$$
\begin{aligned}
\mathcal{H}(\varphi) & =\frac{\pi}{\eta} \sum_{i}\left(\left(\left(p_{i} p_{i}\right)\right)(\varphi)+\left(\left(x_{i}^{\prime} x_{i}^{\prime}\right)\right)(\varphi)\right) \\
& =\lim _{\epsilon \rightarrow 0} \frac{\pi}{\eta}\left(\left.\sum_{i}\left(p_{i}(\tilde{\varphi}) p_{i}(\varphi)+x_{i}^{\prime}(\tilde{\varphi}) x_{i}^{\prime}(\varphi)\right)\right|_{\tilde{\varphi}=\varphi-\epsilon}+2(D-2) \frac{1}{2 \pi \epsilon^{2}}\right) .
\end{aligned}
$$


In the definition of $\zeta$ we also meet the product of $p_{i}$ and $x_{i}^{\prime}$. Their operator product expansion only has singularities of contact type,

$$
p_{i}(\tilde{\varphi}) x_{i}^{\prime}(\varphi)=\frac{i}{2} \partial_{\tilde{\varphi}} \delta(\tilde{\varphi}-\varphi)+\text { regular } .
$$

We therefore get a well-defined composite operator just by point-splitting,

$$
\left(\left(p_{i} x_{i}^{\prime}\right)\right)(\varphi)=\left.\lim _{\epsilon \rightarrow 0}\left(p_{i}(\tilde{\varphi}) x_{i}^{\prime}(\varphi)\right)\right|_{\tilde{\varphi}=\varphi-\epsilon},
$$

and we can define the quantum version of $\zeta$ as

$$
\zeta(\varphi)=\zeta_{0}-\frac{1}{\eta} \int_{0}^{2 \pi} \partial_{\psi} G(\varphi, \psi)\left(\left(\vec{p} \cdot \vec{x}^{\prime}\right)\right)(\psi) d \psi .
$$

When we define the Lorentz generators $M_{i-}$ we also encounter the product of $\mathcal{H}$ and $x_{i}$ as well as the product of $\zeta$ and $p_{i}$, which we have to regularise to obtain well-defined expressions.

Let us start with the product of $\mathcal{H}$ and $x_{i}$. The singularities in the operator product expansion follow via Wick's theorem from the individual contractions of $x_{i}$ and the $x_{j}^{\prime}$ appearing inside $\mathcal{H}$,

$$
\left.x_{i}(\tilde{\varphi}) \mathcal{H}(\varphi)\right|_{\tilde{\varphi}=\varphi-\epsilon}=\frac{\pi}{\eta}\left(-\frac{1}{\pi \epsilon} x_{i}^{\prime}(\varphi)\right)+\text { regular }
$$

Therefore we can define the quantum product of $x_{i}$ and $\mathcal{H}$ by

$$
\left(\left(x_{i} \mathcal{H}\right)\right)(\varphi)=\lim _{\epsilon \rightarrow 0}\left(\left.x_{i}(\tilde{\varphi}) \mathcal{H}(\varphi)\right|_{\tilde{\varphi}=\varphi-\epsilon}+\frac{1}{\epsilon \eta} x_{i}^{\prime}(\varphi)\right) .
$$

A little more work is needed to define the product of $p_{i}$ and $\zeta$, because $\zeta$ is defined as a non-local expression in the fields. The possible singularities come from the contact singularity between $p_{i}$ and $x_{j}^{\prime}$ inside $\zeta$, and from the singularity between $p_{i}$ and the $p_{j}$ inside $\zeta$. The contact singularity is avoided if we consider the symmetrised product $p_{i} \zeta+\zeta p_{i}$, and we find

$$
\frac{1}{2}\left(p_{i}(\tilde{\varphi})\left(\left(\vec{p} \cdot \vec{x}^{\prime}\right)\right)(\psi)+\left(\left(\vec{p} \cdot \vec{x}^{\prime}\right)\right)(\psi) p_{i}(\tilde{\varphi})\right)=-\frac{1}{4 \pi} x_{i}^{\prime}(\psi) \partial_{\psi}\left(\cos \left(\frac{\psi-\tilde{\varphi}}{2}\right) \mathcal{P} \frac{1}{\sin \left(\frac{\psi-\tilde{\varphi}}{2}\right)}\right)+\text { regular }
$$

The possible singularity in the symmetrised product of $\zeta$ and $p_{i}$ is then

$$
\begin{aligned}
\left.\frac{1}{2}\left(p_{i}(\tilde{\varphi}) \zeta(\varphi)+\zeta(\varphi) p_{i}(\tilde{\varphi})\right)\right|_{\tilde{\varphi}=\varphi-\epsilon} \\
\quad=\left.\frac{1}{4 \pi \eta} \int_{0}^{2 \pi} \partial_{\psi} G(\varphi, \psi) x_{i}^{\prime}(\psi) \partial_{\psi}\left(\cos \left(\frac{\psi-\tilde{\varphi}}{2}\right) \mathcal{P} \frac{1}{\sin \left(\frac{\psi-\tilde{\varphi}}{2}\right)}\right)\right|_{\tilde{\varphi}=\varphi-\epsilon} d \psi+\text { regular } \\
\quad=-\frac{1}{4 \pi \eta} \int_{0}^{2 \pi} x_{i}(\psi) \partial_{\psi}\left(\left.\partial_{\psi} G(\varphi, \psi) \partial_{\psi}\left(\cos \left(\frac{\psi-\tilde{\varphi}}{2}\right) \mathcal{P} \frac{1}{\sin \left(\frac{\psi-\tilde{\varphi}}{2}\right)}\right)\right|_{\tilde{\varphi}=\varphi-\epsilon}\right) d \psi+\text { regular } \\
\quad=\partial_{\varphi}\left(\frac{1}{4 \pi \eta} \int_{0}^{2 \pi} x_{i}(\psi)\left(\left.\partial_{\psi} G(\varphi, \psi) \partial_{\psi}\left(\cos \left(\frac{\psi-\tilde{\varphi}}{2}\right) \mathcal{P} \frac{1}{\sin \left(\frac{\psi-\tilde{\varphi}}{2}\right)}\right)\right|_{\tilde{\varphi}=\varphi-\epsilon}\right)\right) d \psi+\text { regular }
\end{aligned}
$$

The possible singular part is therefore a total derivative in $\varphi$, which means that it does not matter in the expression for $M_{i-}$, which involves an integration over $\varphi$. 
Similarly, also the singular part of the product of $x_{i}$ and $\mathcal{H}$ is a total derivative (see (2.13)), which vanishes upon integration. Therefore the quantum definition of $M_{i-}$ using symmetrised products and point-splitting is given by

$$
\begin{aligned}
M_{i-} & =\lim _{\epsilon, \delta \rightarrow 0} M_{i-}(\epsilon, \delta) \\
& =\lim _{\epsilon, \delta \rightarrow 0} \frac{1}{2} \int\left(x_{i}(\varphi+\epsilon) \mathcal{H}(\varphi)+\mathcal{H}(\varphi) x_{i}(\varphi+\epsilon)-\zeta(\varphi+\delta) p_{i}(\varphi)-p_{i}(\varphi) \zeta(\varphi+\delta)\right) d \varphi .
\end{aligned}
$$

This is our starting point for analysing the commutator of $M_{i-}$ and $M_{j-}$.

\section{Basic commutation relations}

To compute the commutators of the Lorentz algebra generators we need to determine the commutators of the fields $x_{i}(\varphi), p_{j}(\varphi), \mathcal{H}(\varphi), \zeta(\varphi)$, which follow from the canonical commutation relations of $x_{i}$ and $p_{j}$, and $\eta$ and $\zeta_{0}$. We first list the results, and present the derivation subsequently. The commutators are

$$
\begin{aligned}
{\left[\eta, \zeta_{0}\right] } & =i \\
{\left[x_{i}(\varphi), p_{j}(\tilde{\varphi})\right] } & =i \delta_{i j} \delta(\varphi-\tilde{\varphi}) \\
{\left[\mathcal{H}(\varphi), p_{j}(\tilde{\varphi})\right] } & =\frac{2 \pi i}{\eta} \partial_{\varphi} \delta(\varphi-\tilde{\varphi}) x_{j}^{\prime}(\varphi) \\
{\left[x_{i}(\varphi), \zeta(\tilde{\varphi})\right] } & =-\frac{i}{\eta} \partial_{\varphi} G(\tilde{\varphi}, \varphi) x_{i}^{\prime}(\varphi) \\
{[\mathcal{H}(\varphi), \zeta(\tilde{\varphi})] } & =-\frac{2 \pi i}{\eta} \delta(\varphi-\tilde{\varphi}) \mathcal{H}(\varphi)-\frac{i}{\eta} \partial_{\varphi}\left(\partial_{\varphi} G(\tilde{\varphi}, \varphi) \mathcal{H}(\varphi)\right)+\frac{\pi i}{3 \eta^{2}}(D-2) \partial_{\varphi}^{2} \delta(\varphi-\tilde{\varphi}) \\
{[\mathcal{H}(\varphi), \mathcal{H}(\tilde{\varphi})] } & =\frac{2 i \pi^{2}}{\eta^{2}} \partial_{\varphi} \delta(\varphi-\tilde{\varphi})\left(\vec{p}(\varphi) \cdot \vec{x}^{\prime}(\tilde{\varphi})+\vec{x}^{\prime}(\tilde{\varphi}) \cdot \vec{p}(\varphi)+\vec{p}(\tilde{\varphi}) \cdot \vec{x}^{\prime}(\varphi)+\vec{x}^{\prime}(\varphi) \cdot \vec{p}(\tilde{\varphi})\right) \\
{\left[\mathcal{H}(\varphi), x_{j}(\tilde{\varphi})\right] } & =-\frac{2 \pi i}{\eta} \delta(\varphi-\tilde{\varphi}) p_{j}(\tilde{\varphi}) \\
{\left[\zeta(\varphi), p_{j}(\tilde{\varphi})\right] } & =\frac{i}{\eta} \partial_{\tilde{\varphi}}\left(\partial_{\tilde{\varphi}} G(\varphi, \tilde{\varphi}) p_{j}(\tilde{\varphi})\right) \\
{[\zeta(\varphi), \zeta(\tilde{\varphi})] } & =-\frac{i}{\eta} \partial_{\varphi} G(\tilde{\varphi}, \varphi)\left(\zeta^{\prime}(\varphi)+\zeta^{\prime}(\tilde{\varphi})\right) .
\end{aligned}
$$

The derivation is straightforward:

- (3.1), (3.2): These are the canonical commutation relations.

- (3.3): We use the definition of $\mathcal{H}$ via point-splitting (see (2.9)). The only contribution to the commutator comes from the $x^{2}$ term in $\mathcal{H}$,

$$
\begin{aligned}
{\left[\mathcal{H}(\varphi), p_{j}(\tilde{\varphi})\right] } & =\frac{\pi}{\eta} \lim _{\epsilon \rightarrow 0}\left[\vec{x}^{\prime}(\varphi) \cdot \vec{x}^{\prime}(\varphi-\epsilon), p_{j}(\tilde{\varphi})\right] \\
& =i \frac{\pi}{\eta} \lim _{\epsilon \rightarrow 0}\left(x_{j}^{\prime}(\varphi) \partial_{\varphi} \delta(\varphi-\tilde{\varphi}-\epsilon)+x_{j}^{\prime}(\varphi+\epsilon) \partial_{\varphi} \delta(\varphi-\tilde{\varphi})\right)=\frac{2 \pi i}{\eta} \partial_{\varphi} \delta(\varphi-\tilde{\varphi}) x_{j}^{\prime}(\varphi) .
\end{aligned}
$$

- (3.4):

$$
\left[x_{i}(\varphi), \zeta(\tilde{\varphi})\right]=\lim _{\epsilon \rightarrow 0} \frac{1}{\eta} \int G(\tilde{\varphi}, \psi) \partial_{\psi}\left[x_{i}(\varphi), \vec{p}(\psi) \cdot \vec{x}^{\prime}(\psi-\epsilon)\right] d \psi=-\frac{i}{\eta} \partial_{\varphi} G(\tilde{\varphi}, \varphi) x_{i}^{\prime}(\varphi)
$$


where in the second step we integrated by parts, computed the commutator $\left[x_{i}(\varphi), \vec{p}(\psi) \cdot \vec{x}^{\prime}(\psi-\epsilon)\right]$ and took the limit $\epsilon \rightarrow 0$.

- (3.5): The derivation of the commutator of $\mathcal{H}$ and $\zeta$ is straightforward, but slightly more involved,

$$
\begin{aligned}
& {\left.[\mathcal{H}(\varphi), \zeta(\tilde{\varphi})]=\left[\frac{\pi}{\eta}\left(((\vec{p} \cdot \vec{p}))(\varphi)+\left(\vec{x}^{\prime} \cdot \vec{x}^{\prime}\right)\right)(\varphi)\right), \zeta_{0}-\frac{1}{\eta} \int \partial_{\psi} G(\tilde{\varphi}, \psi)\left(\left(\vec{p} \cdot \vec{x}^{\prime}\right)\right)(\psi) d \psi\right] } \\
&=- \frac{i \pi}{\eta^{2}}\left(((\vec{p} \cdot \vec{p}))(\varphi)+\left(\left(\vec{x}^{\prime} \cdot \vec{x}^{\prime}\right)\right)(\varphi)\right) \\
&-\frac{\pi}{\eta^{2}} \lim _{\epsilon, \tilde{\epsilon} \rightarrow 0} \int \partial_{\psi} G(\tilde{\varphi}, \psi)\left[\vec{p}(\varphi) \cdot \vec{p}(\varphi-\epsilon)+\vec{x}^{\prime}(\varphi) \cdot \vec{x}^{\prime}(\varphi-\epsilon), \vec{p}(\psi) \cdot \vec{x}^{\prime}(\psi-\tilde{\epsilon})\right] d \psi \\
&=- \frac{i}{\eta} \mathcal{H}(\varphi)+\frac{2 \pi i}{\eta^{2}} \lim _{\tilde{\epsilon} \rightarrow 0} \int \partial_{\psi} G(\tilde{\varphi}, \psi)\left(\partial_{\psi} \delta(\varphi-\psi+\tilde{\epsilon}) \vec{p}(\varphi) \cdot \vec{p}(\psi)+\partial_{\psi} \delta(\varphi-\psi) \vec{x}^{\prime}(\varphi) \cdot \vec{x}^{\prime}(\psi-\tilde{\epsilon})\right) d \psi \\
&=- \frac{i}{\eta} \mathcal{H}(\varphi)+\frac{2 \pi i}{\eta^{2}} \int \partial_{\psi} G(\tilde{\varphi}, \psi) \partial_{\psi} \delta(\varphi-\psi)\left(\vec{p}(\varphi) \cdot \vec{p}(\psi)+\vec{x}^{\prime}(\varphi) \cdot \vec{x}^{\prime}(\psi)-2(D-2) S_{\operatorname{sing}}(\varphi, \psi)\right) d \psi \\
&+\frac{2 \pi i}{\eta^{2}}(D-2) \lim _{\tilde{\epsilon} \rightarrow 0} \int \partial_{\psi} G(\tilde{\varphi}, \psi)\left(\partial_{\psi} \delta(\varphi-\psi) S_{\operatorname{sing}}(\varphi, \psi-\tilde{\epsilon})+\partial_{\psi} \delta(\varphi-\psi+\tilde{\epsilon}) S_{\operatorname{sing}}(\varphi, \psi)\right) d \psi \\
&=- \frac{i}{\eta} \mathcal{H}(\varphi)-\frac{2 \pi i}{\eta^{2}}\left(\partial_{\varphi}^{2} G(\tilde{\varphi}, \varphi)\left(\frac{\eta}{\pi} \mathcal{H}(\varphi)+\frac{D-2}{12 \pi}\right)+\partial_{\varphi} G(\tilde{\varphi}, \varphi) \frac{\eta}{2 \pi} \partial_{\varphi} \mathcal{H}(\varphi)\right) \\
&-\frac{2 \pi i}{\eta^{2}}(D-2) \int \partial_{\psi} G(\tilde{\varphi}, \psi) \frac{1}{12 \pi}\left(\partial_{\psi} \delta(\varphi-\psi)+\partial_{\psi}^{3} \delta(\varphi-\psi)\right) d \psi \\
&=-\frac{2 \pi i}{\eta} \delta(\varphi-\tilde{\varphi}) \mathcal{H}(\varphi)-\frac{i}{\eta} \partial_{\varphi}\left(\partial_{\varphi} G(\tilde{\varphi}, \varphi) \mathcal{H}(\varphi)\right)+\frac{\pi i}{3 \eta^{2}}(D-2) \partial_{\varphi}^{2} \delta(\varphi-\tilde{\varphi})
\end{aligned}
$$

where we used that

$$
\begin{aligned}
\partial_{\psi} \delta(\varphi-\psi) S_{\text {sing }}(\varphi, \psi-\tilde{\epsilon}) & =\partial_{\psi} \delta(\varphi-\psi) S_{\text {sing }}(\varphi, \varphi-\tilde{\epsilon})-\left.\delta(\varphi-\psi) \partial_{\psi} S_{\operatorname{sing}}(\varphi, \psi-\tilde{\epsilon})\right|_{\psi=\varphi} \\
& =\left(-\frac{1}{2 \pi \tilde{\epsilon}^{2}}-\frac{1}{24 \pi}+\mathcal{O}\left(\tilde{\epsilon}^{2}\right)\right) \partial_{\psi} \delta(\varphi-\psi)+\left(\frac{1}{\pi \tilde{\epsilon}^{3}}+\mathcal{O}(\tilde{\epsilon})\right) \delta(\varphi-\psi) .
\end{aligned}
$$

Note that the commutator of $\mathcal{H}$ and $\zeta$ contains a term that depends on the number $D$ of space-time dimensions.

- (3.6):

$$
\begin{aligned}
{[\mathcal{H}(\varphi), \mathcal{H}(\tilde{\varphi})] } & =\frac{\pi^{2}}{\eta^{2}} \lim _{\epsilon, \tilde{\epsilon} \rightarrow 0}\left[\vec{p}(\varphi) \cdot \vec{p}(\varphi-\epsilon)+\vec{x}^{\prime}(\varphi) \cdot \vec{x}^{\prime}(\varphi-\epsilon), \vec{p}(\tilde{\varphi}) \cdot \vec{p}(\tilde{\varphi}-\tilde{\epsilon})+\vec{x}^{\prime}(\tilde{\varphi}) \cdot \vec{x}^{\prime}(\tilde{\varphi}-\tilde{\epsilon})\right] \\
& =\frac{2 i \pi^{2}}{\eta^{2}} \partial_{\varphi} \delta(\varphi-\tilde{\varphi})\left(\vec{p}(\varphi) \cdot \vec{x}^{\prime}(\tilde{\varphi})+\vec{x}^{\prime}(\tilde{\varphi}) \cdot \vec{p}(\varphi)+\vec{p}(\tilde{\varphi}) \cdot \vec{x}^{\prime}(\varphi)+\vec{x}^{\prime}(\varphi) \cdot \vec{p}(\tilde{\varphi})\right)
\end{aligned}
$$

- (3.7):

$$
\begin{aligned}
{\left[\mathcal{H}(\varphi), x_{j}(\tilde{\varphi})\right] } & =\frac{\pi}{\eta} \lim _{\epsilon \rightarrow 0}\left[\vec{p}(\varphi) \cdot \vec{p}(\varphi-\epsilon), x_{j}(\tilde{\varphi})\right] \\
& =-i \frac{\pi}{\eta} \lim _{\epsilon \rightarrow 0}\left(p_{j}(\varphi) \delta(\varphi-\tilde{\varphi}-\epsilon)+p_{j}(\varphi+\epsilon) \delta(\varphi-\tilde{\varphi})\right)=-\frac{2 \pi i}{\eta} \delta(\varphi-\tilde{\varphi}) p_{j}(\varphi)
\end{aligned}
$$


- (3.8):

$$
\left[\zeta(\varphi), p_{j}(\tilde{\varphi})\right]=-\lim _{\epsilon \rightarrow 0} \frac{1}{\eta} \int G(\varphi, \psi) \partial_{\psi}\left[p_{j}(\tilde{\varphi}), \vec{p}(\psi) \cdot \vec{x}^{\prime}(\psi-\epsilon)\right] d \psi=\frac{i}{\eta} \partial_{\tilde{\varphi}}\left(\partial_{\tilde{\varphi}} G(\varphi, \tilde{\varphi}) p_{j}(\tilde{\varphi})\right)
$$

where in the second equality we integrated by parts, computed the commutator $\left[p_{j}(\tilde{\varphi}), \vec{p}(\psi) \cdot \vec{x}^{\prime}(\psi-\epsilon)\right]$, integrated by parts again and finally took the limit $\epsilon \rightarrow 0$.

- (3.9):

$$
\begin{aligned}
{[\zeta(\varphi), \zeta(\tilde{\varphi})]=} & \frac{i}{\eta}(\zeta(\varphi)-\zeta(\tilde{\varphi})) \\
& +\frac{1}{4 \eta^{2}} \lim _{\epsilon, \tilde{\epsilon} \rightarrow 0} \iint \partial_{\tilde{\psi}} G(\tilde{\varphi}, \tilde{\psi}) \partial_{\psi} G(\varphi, \psi)\left[\vec{p}(\psi) \cdot \vec{x}^{\prime}(\psi-\epsilon), \vec{p}(\tilde{\psi}) \cdot \vec{x}^{\prime}(\tilde{\psi}-\tilde{\epsilon})\right] d \psi d \tilde{\psi} \\
= & \lim _{\epsilon, \tilde{\epsilon} \rightarrow 0}\left(-\frac{2 \pi}{\eta^{2}} \partial_{\varphi} G(\tilde{\varphi}, \varphi) \vec{p}(\varphi) \cdot \vec{x}^{\prime}(\varphi-\epsilon-\tilde{\epsilon})-(\varphi \leftrightarrow \tilde{\varphi})\right) \\
= & -\frac{i}{\eta} \partial_{\varphi} G(\tilde{\varphi}, \varphi)\left(\zeta^{\prime}(\varphi)+\zeta^{\prime}(\tilde{\varphi})\right),
\end{aligned}
$$

where in the last step we used that $\zeta^{\prime}=\frac{2 \pi}{\eta}\left(\left(\vec{p} \cdot \vec{x}^{\prime}\right)\right)$.

\section{The crucial commutator}

We now want to analyse the commutator of the generators $M_{i-}$ and $M_{j-}$, which we defined in (2.20). It can be decomposed as

$$
\begin{aligned}
& {\left[M_{i-}, M_{j-}\right]=} \lim _{\epsilon, \delta \rightarrow 0} \lim _{\tilde{\epsilon}, \tilde{\delta} \rightarrow 0}\left[M_{i-}(\epsilon, \delta), M_{i-}(\tilde{\epsilon}, \tilde{\delta})\right] \\
&=\lim _{\epsilon, \delta \rightarrow 0} \lim _{\tilde{\epsilon}, \tilde{\delta} \rightarrow 0} \iint\left[x_{i}(\varphi+\epsilon) \mathcal{H}(\varphi)-\frac{1}{2}\left(\zeta(\varphi+\delta) p_{i}(\varphi)+p_{i}(\varphi) \zeta(\varphi+\delta)\right),\right. \\
&\left.\quad x_{j}(\tilde{\varphi}+\tilde{\epsilon}) \mathcal{H}(\tilde{\varphi})-\frac{1}{2}\left(\zeta(\tilde{\varphi}+\tilde{\delta}) p_{j}(\tilde{\varphi})+p_{j}(\tilde{\varphi}) \zeta(\tilde{\varphi}+\tilde{\delta})\right)\right] d \varphi d \tilde{\varphi} .
\end{aligned}
$$

Here we used the fact that $x_{i}(\varphi+\epsilon)$ and $\mathcal{H}(\varphi)$ commute for $\epsilon>0$ (see (3.7)). There are four types of contributions: the commutator of the terms of the form $x \mathcal{H}$, the two mixed commutators of $x \mathcal{H}$ and $\zeta p$, and the commutator of the terms of the form $\zeta p$.

At the end we want to analyse the behaviour when $\epsilon, \delta$ and $\tilde{\epsilon}, \tilde{\delta}$ go to zero. It is not guaranteed that this limit exists, and indeed we will see that e.g. the commutator of the terms of the form $x \mathcal{H}$ alone is singular when the regularisation parameters go to zero; this singularity will go away when we combine all contributions to the commutator of $M_{i-}$ and $M_{j-}$. On the other hand we expect that we can take one set of parameters to zero without getting a singularity in the different contributions: because the individual entries entering the commutator are regularised and do not show any singularity in $\epsilon, \delta$ or in $\tilde{\epsilon}, \tilde{\delta}$, the only way a new singularity can appear is through terms that become singular when both type of parameters go to zero (like $\left.(\epsilon+\tilde{\epsilon})^{-1}\right)$.

Our strategy will therefore be to always take the limit $\tilde{\epsilon}, \tilde{\delta}$ to zero first, and then consider the limit when $\epsilon$ and $\delta$ go to zero.

We start by analysing the commutator of the terms $x \mathcal{H}$ in detail. We then present the results for the remaining commutators, and evaluate the total expression. 


\subsection{Commutators of the form $\left[x_{i} \mathcal{H}, x_{j} \mathcal{H}\right]$}

We want to analyse

$$
C_{i j}^{x \mathcal{H}, x \mathcal{H}}(\epsilon, \tilde{\epsilon})=\iint\left[x_{i}(\varphi+\epsilon) \mathcal{H}(\varphi), x_{j}(\tilde{\varphi}+\tilde{\epsilon}) \mathcal{H}(\tilde{\varphi})\right] d \varphi d \tilde{\varphi}
$$

by using the commutation relations that we worked out in section 3 . In a first step we obtain

$$
\begin{aligned}
C_{i j}^{x \mathcal{H}, x \mathcal{H}}(\epsilon, \tilde{\epsilon})=\frac{2 \pi i}{\eta} \int( & x_{j}(\varphi+\tilde{\epsilon}) p_{i}(\varphi) \mathcal{H}(\varphi-\epsilon)-x_{i}(\varphi+\epsilon+\tilde{\epsilon}) p_{j}(\varphi+\tilde{\epsilon}) \mathcal{H}(\varphi) \\
& \left.+\left(x_{i}(\varphi+\epsilon) x_{j}^{\prime}(\varphi+\tilde{\epsilon})-x_{i}^{\prime}(\varphi+\epsilon) x_{j}(\varphi+\tilde{\epsilon})\right) \zeta^{\prime}(\varphi)\right) d \varphi
\end{aligned}
$$

We now consider the behaviour when $\tilde{\epsilon}$ goes to zero, and we find

$$
\begin{aligned}
C_{i j}^{x \mathcal{H}, x \mathcal{H}}(\epsilon, \tilde{\epsilon})=\frac{2 \pi i}{\eta} \int & \left\{\left(\left(x_{j} p_{i}\right)\right)(\varphi) \mathcal{H}(\varphi-\epsilon)\right. \\
& -\left(x_{i}(\varphi+\epsilon)+\tilde{\epsilon} x_{i}^{\prime}(\varphi+\epsilon)+\frac{1}{2} \tilde{\epsilon}^{2} x_{i}^{\prime \prime}(\varphi+\epsilon)\right)\left(\left(\left(p_{j} \mathcal{H}\right)\right)(\varphi)-\frac{1}{\eta \tilde{\epsilon}^{2}} p_{j}(\varphi)\right) \\
& \left.+x_{i}(\varphi+\epsilon)\left(\left(\left(x_{j}^{\prime} \zeta^{\prime}\right)\right)(\varphi)-\frac{1}{\eta \tilde{\epsilon}^{2}} p_{j}(\varphi)\right)-x_{i}^{\prime}(\varphi+\epsilon)\left(\left(\left(x_{j} \zeta^{\prime}\right)\right)(\varphi)+\frac{1}{\eta \tilde{\epsilon}} p_{j}(\varphi)\right)\right\} d \varphi+\mathcal{O}(\tilde{\epsilon}) \\
=\frac{2 \pi i}{\eta} \int & \left\{\left(\left(x_{j} p_{i}\right)\right)(\varphi+\epsilon) \mathcal{H}(\varphi)-x_{i}(\varphi+\epsilon)\left(\left(p_{j} \mathcal{H}\right)\right)(\varphi)\right. \\
& \left.+x_{i}(\varphi+\epsilon)\left(\left(x_{j}^{\prime} \zeta^{\prime}\right)\right)(\varphi)-x_{i}^{\prime}(\varphi+\epsilon)\left(\left(x_{j} \zeta^{\prime}\right)\right)(\varphi)+\frac{1}{2 \eta} x_{i}^{\prime \prime}(\varphi+\epsilon) p_{j}(\varphi)\right\} d \varphi+\mathcal{O}(\tilde{\epsilon})
\end{aligned}
$$

where in the first summand we shifted the integration variable by $\epsilon$. As expected there is no singularity when $\tilde{\epsilon}$ is taken to zero.

We now want to analyse the possible singularities in $\epsilon$. The singularities between normal-ordered expressions arise from singularities between the constituents, we have e.g.

$$
\begin{aligned}
x_{i}(\varphi+\epsilon)\left(\left(x_{j}^{\prime} \zeta^{\prime}\right)\right)(\varphi) & =\frac{2 \pi}{\eta} x_{i}(\varphi+\epsilon)\left(\left(x_{j}^{\prime}\left(\left(p \cdot x^{\prime}\right)\right)\right)\right)(\varphi) \\
& =\frac{1}{\eta \epsilon}\left(\left(x_{j}^{\prime} p_{i}\right)\right)(\varphi)+\text { regular } .
\end{aligned}
$$

OPE normal ordering is in general not associative, and we want to define the normal-ordering of several operators in a right-nested way,

$$
((A B C)):=((A((B C)))) .
$$

In the case at hand we have (see appendix $\mathrm{C}$ )

$$
\begin{aligned}
& \left.\left(\left(\left(x_{j} p_{i}\right)\right) \mathcal{H}\right)\right)=\left(\left(x_{j} p_{i} \mathcal{H}\right)\right)-\frac{1}{2 \eta}\left(\left(\left(x_{j}^{\prime \prime} p_{i}\right)\right)-2\left(\left(p_{i}^{\prime} x_{j}^{\prime}\right)\right)\right) \\
& \left.\left(\left(\left(x_{i} x_{j}^{\prime}\right)\right) \zeta^{\prime}\right)\right)=\left(\left(x_{i} x_{j}^{\prime} \zeta^{\prime}\right)\right)+\frac{1}{2 \eta}\left(2\left(\left(x_{j}^{\prime \prime} p_{i}\right)\right)-\left(\left(x_{i}^{\prime \prime} p_{j}\right)\right)\right),
\end{aligned}
$$


so that we find

$$
\begin{aligned}
C_{i j}^{x \mathcal{H}, x \mathcal{H}}(\epsilon, 0)=\frac{2 \pi i}{\eta} \int\left\{\left(\left(\left(\left(x_{j} p_{i}\right)\right) \mathcal{H}\right)\right)(\varphi)-\frac{1}{\epsilon^{2} \eta}\left(\left(p_{i} x_{j}\right)\right)(\varphi)\right)-\left(\left(\left(x_{i} p_{j} \mathcal{H}\right)\right)(\varphi)+\frac{1}{\epsilon \eta}\left(\left(p_{j} x_{i}^{\prime}\right)\right)(\varphi)\right) \\
\left.+\left(\left(\left(x_{i} x_{j}^{\prime} \zeta^{\prime}\right)\right)(\varphi)+\frac{1}{\epsilon \eta}\left(\left(x_{j}^{\prime} p_{i}\right)\right)(\varphi)\right)-\left(\left(\left(x_{i}^{\prime} x_{j} \zeta^{\prime}\right)\right)(\varphi)-\frac{1}{\epsilon^{2} \eta}\left(\left(x_{j} p_{i}\right)\right)(\varphi)\right)+\frac{1}{2 \eta}\left(\left(x_{i}^{\prime \prime} p_{j}\right)\right)(\varphi)\right\} d \varphi+\mathcal{O}(\epsilon) \\
=\frac{2 \pi i}{\eta} \int\left\{\frac{1}{\epsilon \eta}\left(\left(\left(p_{i} x_{j}^{\prime}\right)\right)(\varphi)-\left(\left(p_{j} x_{i}^{\prime}\right)\right)(\varphi)\right)+\frac{1}{2 \eta}\left(\left(x_{i}^{\prime \prime} p_{j}\right)\right)(\varphi)-\frac{3}{2 \eta}\left(\left(x_{j}^{\prime \prime} p_{i}\right)\right)(\varphi)\right. \\
\left.+\left(\left(x_{j} p_{i} \mathcal{H}\right)\right)(\varphi)-\left(\left(x_{i} p_{j} \mathcal{H}\right)\right)(\varphi)+\left(\left(x_{i} x_{j}^{\prime} \zeta^{\prime}\right)\right)(\varphi)-\left(\left(x_{i}^{\prime} x_{j} \zeta^{\prime}\right)\right)(\varphi)\right\} d \varphi+\mathcal{O}(\epsilon)
\end{aligned}
$$

The remaining singularity in $\epsilon$ will be cancelled by the contribution $C_{i j}^{x \mathcal{H}, p \zeta}$ that we discuss in the following.

\subsection{Commutators of the form $\left[x_{i} \mathcal{H}, p_{j} \zeta\right]$}

We now turn to the analysis of the contribution

$$
C_{i j}^{x \mathcal{H}, p \zeta}(\epsilon, \tilde{\delta})=-\frac{1}{2} \iint\left[x_{i}(\varphi+\epsilon) \mathcal{H}(\varphi), \zeta(\tilde{\varphi}+\tilde{\delta}) p_{j}(\tilde{\varphi})+p_{j}(\tilde{\varphi}) \zeta(\tilde{\varphi}+\tilde{\delta})\right] d \varphi d \tilde{\varphi}
$$

Evaluating the commutator we find (for $i \neq j$ )

$$
\begin{aligned}
C_{i j}^{x \mathcal{H}, p \zeta}(\epsilon, \tilde{\delta})= & \frac{2 \pi i}{\eta} \int\left(x_{i}(\varphi+\epsilon) p_{j}(\varphi-\tilde{\delta}) \mathcal{H}(\varphi)-x_{i}(\varphi+\epsilon-\tilde{\delta}) x_{j}^{\prime}(\varphi-\tilde{\delta}) \zeta^{\prime}(\varphi)\right) d \varphi \\
& +\frac{i}{\eta} \iint x_{i}^{\prime}(\varphi+\epsilon) p_{j}(\tilde{\varphi}) \mathcal{H}(\varphi) \partial_{\varphi}(G(\tilde{\varphi}+\tilde{\delta}, \varphi+\epsilon)-G(\tilde{\varphi}+\tilde{\delta}, \varphi)) d \varphi d \tilde{\varphi} \\
& -\frac{\pi i}{3 \eta^{2}}(D-2) \int x_{i}^{\prime \prime}(\varphi+\tilde{\delta}+\epsilon) p_{j}(\varphi) d \varphi
\end{aligned}
$$

We expand this expression first in $\tilde{\delta}$, and we obtain

$$
\begin{aligned}
C_{i j}^{x \mathcal{H}, p \zeta}(\epsilon, \tilde{\delta})= & \frac{2 \pi i}{\eta} \int\left(x_{i}(\varphi+\epsilon)\left(\left(\left(p_{j} \mathcal{H}\right)\right)(\varphi)-\frac{1}{\tilde{\delta}^{2} \eta} p_{j}(\varphi)\right)\right. \\
& \left.-\left(x_{i}(\varphi+\epsilon)-\tilde{\delta} x_{i}^{\prime}(\varphi+\epsilon)+\frac{\tilde{\delta}^{2}}{2} x_{i}^{\prime \prime}(\varphi+\epsilon)\right)\left(\left(\left(x_{j}^{\prime} \zeta^{\prime}\right)\right)(\varphi)-\frac{1}{\tilde{\delta}^{2} \eta} p_{j}(\varphi)\right)\right) d \varphi \\
+ & \frac{i}{\eta} \iint x_{i}^{\prime}(\varphi+\epsilon)\left(p_{j}(\tilde{\varphi}) \mathcal{H}(\varphi)-\frac{2 \pi}{\eta} S_{\operatorname{sing}}(\tilde{\varphi}, \varphi) p_{j}(\varphi)\right) \partial_{\varphi}(G(\tilde{\varphi}+\tilde{\delta}, \varphi+\epsilon)-G(\tilde{\varphi}+\tilde{\delta}, \varphi)) d \varphi d \tilde{\varphi} \\
+ & \frac{i}{\eta} \iint x_{i}^{\prime}(\varphi+\epsilon)\left(\frac{2 \pi}{\eta} S_{\operatorname{sing}}(\tilde{\varphi}, \varphi) p_{j}(\varphi)\right) \partial_{\varphi}(G(\tilde{\varphi}+\tilde{\delta}, \varphi+\epsilon)-G(\tilde{\varphi}+\tilde{\delta}, \varphi)) d \varphi d \tilde{\varphi} \\
& -\frac{\pi i}{3 \eta^{2}}(D-2) \int x_{i}^{\prime \prime}(\varphi+\epsilon) p_{j}(\varphi) d \varphi+\mathcal{O}(\tilde{\delta}) .
\end{aligned}
$$


In the third and fourth line we have subtracted and added the singular piece of $p_{j}(\tilde{\varphi}) \mathcal{H}(\varphi)$. In the third line, there is therefore no singularity coming from the operator part when $\varphi$ and $\tilde{\varphi}$ are close together, and we can just set $\tilde{\delta}$ to 0 . The fourth line can be evaluated by writing $S_{\text {sing }}$ as a derivative and then using partial integration,

$$
\begin{aligned}
& \frac{i}{\eta} \iint x_{i}^{\prime}(\varphi+\epsilon)\left(\frac{2 \pi}{\eta} S_{\operatorname{sing}}(\tilde{\varphi}, \varphi) p_{j}(\varphi)\right) \partial_{\varphi}(G(\tilde{\varphi}+\tilde{\delta}, \varphi+\epsilon)-G(\tilde{\varphi}+\tilde{\delta}, \varphi)) d \varphi d \tilde{\varphi} \\
& \quad=\frac{i}{2 \eta^{2}} \iint x_{i}^{\prime}(\varphi+\epsilon) \partial_{\tilde{\varphi}}\left(\cos \frac{\tilde{\varphi}-\varphi}{2} \mathcal{P} \frac{1}{\sin \frac{\tilde{\varphi}-\varphi}{2}}\right) p_{j}(\varphi) \partial_{\varphi}(G(\tilde{\varphi}+\tilde{\delta}, \varphi+\epsilon)-G(\tilde{\varphi}+\tilde{\delta}, \varphi)) d \varphi d \tilde{\varphi} \\
& \quad=-\frac{i}{2 \eta^{2}} \iint x_{i}^{\prime}(\varphi+\epsilon)\left(\cos \frac{\tilde{\varphi}-\varphi}{2} \mathcal{P} \frac{1}{\sin \frac{\tilde{\varphi}-\varphi}{2}}\right) p_{j}(\varphi)(-2 \pi \delta(\tilde{\varphi}-\varphi+\tilde{\delta}-\epsilon)+2 \pi \delta(\tilde{\varphi}-\varphi+\tilde{\delta})) d \varphi d \tilde{\varphi} \\
& \quad=\frac{i \pi}{\eta^{2}} \int x_{i}^{\prime}(\varphi+\epsilon) p_{j}(\varphi)\left(\cos \frac{\epsilon-\tilde{\delta}}{2} \frac{1}{\sin \frac{\epsilon-\tilde{\delta}}{2}}+\cos \frac{\tilde{\delta}}{2} \frac{1}{\sin \frac{\tilde{\delta}}{2}}\right) d \varphi \\
& \quad=\frac{i \pi}{\eta^{2}} \int x_{i}^{\prime}(\varphi+\epsilon) p_{j}(\varphi)\left(\cos \frac{\epsilon}{2} \frac{1}{\sin \frac{\epsilon}{2}}+\frac{2}{\tilde{\delta}}\right) d \varphi+\mathcal{O}(\tilde{\delta}) .
\end{aligned}
$$

Inserting this result into (4.15) we obtain

$$
\begin{aligned}
C_{i j}^{x \mathcal{H}, p \zeta}(\epsilon, \tilde{\delta})= & \frac{2 \pi i}{\eta} \int\left(x_{i}(\varphi+\epsilon)\left(\left(p_{j} \mathcal{H}\right)\right)(\varphi)-x_{i}(\varphi+\epsilon)\left(\left(x_{j}^{\prime} \zeta^{\prime}\right)\right)(\varphi)+\frac{1}{2 \eta} x_{i}^{\prime \prime}(\varphi+\epsilon) p_{j}(\varphi)\right) d \varphi \\
& +\frac{i}{\eta} \iint x_{i}^{\prime}(\varphi+\epsilon)\left(p_{j}(\tilde{\varphi}) \mathcal{H}(\varphi)-\frac{2 \pi}{\eta} S_{\operatorname{sing}}(\tilde{\varphi}, \varphi) p_{j}(\varphi)\right) \partial_{\varphi}(G(\tilde{\varphi}, \varphi+\epsilon)-G(\tilde{\varphi}, \varphi)) d \varphi d \tilde{\varphi} \\
& +\frac{i \pi}{\eta^{2}} \int x_{i}^{\prime}(\varphi+\epsilon) p_{j}(\varphi)\left(\cos \frac{\epsilon}{2} \frac{1}{\sin \frac{\epsilon}{2}}\right) d \varphi \\
& -\frac{\pi i}{3 \eta^{2}}(D-2) \int x_{i}^{\prime \prime}(\varphi+\epsilon) p_{j}(\varphi) d \varphi+\mathcal{O}(\tilde{\delta})
\end{aligned}
$$

We observe that the expression contains no singularity in $\tilde{\delta}$. Now we expand in $\epsilon$,

$$
\begin{aligned}
C_{i j}^{x \mathcal{H}, p \zeta}(\epsilon, 0)= & \frac{2 \pi i}{\eta} \int\left(\left(\left(x_{i} p_{j} \mathcal{H}\right)\right)(\varphi)+\frac{1}{\epsilon \eta}\left(\left(p_{j} x_{i}^{\prime}\right)\right)(\varphi)-\left(\left(x_{i} x_{j}^{\prime} \zeta^{\prime}\right)\right)(\varphi)-\frac{1}{\epsilon \eta}\left(\left(p_{i} x_{j}^{\prime}\right)\right)(\varphi)+\frac{1}{2 \eta}\left(\left(x_{i}^{\prime \prime} p_{j}\right)\right)(\varphi)\right) d \varphi \\
& +\frac{i}{\eta} \int\left(-\frac{2 \pi}{\epsilon \eta}\left(\left(x_{i}^{\prime} p_{j}\right)\right)(\varphi)+\frac{\pi}{\eta}\left(\left(x_{i}^{\prime \prime} p_{j}\right)\right)(\varphi)\right) d \varphi \\
& +\frac{i \pi}{\eta^{2}} \int\left(\frac{2}{\epsilon}\left(\left(x_{i}^{\prime} p_{j}\right)\right)(\varphi)+2\left(\left(x_{i}^{\prime \prime} p_{j}\right)\right)(\varphi)\right) d \varphi \\
& -\frac{\pi i}{3 \eta^{2}}(D-2) \int\left(\left(x_{i}^{\prime \prime} p_{j}\right)\right)(\varphi) d \varphi+\mathcal{O}(\epsilon) \\
= & \frac{2 \pi i}{\eta} \int\left\{\frac{1}{\epsilon \eta}\left(\left(\left(p_{j} x_{i}^{\prime}\right)\right)(\varphi)-\left(\left(p_{i} x_{j}^{\prime}\right)\right)(\varphi)\right)+\left(\left(x_{i} p_{j} \mathcal{H}\right)\right)(\varphi)-\left(\left(x_{i} x_{j}^{\prime} \zeta^{\prime}\right)\right)(\varphi)\right. \\
& \left.+\frac{2}{\eta}\left(\left(x_{i}^{\prime \prime} p_{j}\right)\right)(\varphi)-\frac{D-2}{6 \eta}\left(\left(x_{i}^{\prime \prime} p_{j}\right)\right)(\varphi)\right\} d \varphi+\mathcal{O}(\epsilon) .
\end{aligned}
$$


The remaining singularity cancels the terms that we found in $C_{i j}^{x \mathcal{H}, x \mathcal{H}}$ (see (4.12)), so that we find

$$
\begin{aligned}
\lim _{\epsilon \rightarrow 0} \int & {\left[x_{i}(\varphi+\epsilon) \mathcal{H}(\varphi), M_{j-}\right] d \varphi } \\
= & \lim _{\epsilon \rightarrow 0}\left(C_{i j}^{x \mathcal{H}, x \mathcal{H}}(\epsilon, 0)+C_{i j}^{x \mathcal{H}, p \zeta}(\epsilon, 0)\right) \\
= & \frac{2 \pi i}{\eta} \int\left\{\left(\left(x_{j} p_{i} \mathcal{H}\right)\right)(\varphi)-\left(\left(x_{i}^{\prime} x_{j} \zeta^{\prime}\right)\right)(\varphi)+\frac{5}{2 \eta}\left(\left(x_{i}^{\prime \prime} p_{j}\right)\right)(\varphi)-\frac{3}{2 \eta}\left(\left(x_{j}^{\prime \prime} p_{i}\right)\right)(\varphi)-\frac{D-2}{6 \eta}\left(\left(x_{i}^{\prime \prime} p_{j}\right)\right)(\varphi)\right\} d \varphi .
\end{aligned}
$$

\subsection{Remaining commutators and final result}

The remaining commutators can be computed analogously to the computations we displayed above, which is done in the appendices $\mathrm{A}$ and $\mathrm{B}$, and the results are given in $\mathrm{A} .5$ and $(\mathrm{B} .10)$. Their sum does not contain any singularity, and one obtains

$$
\begin{aligned}
\lim _{\delta \rightarrow 0} \int & {\left[-\frac{1}{2}\left(\zeta(\varphi+\delta) p_{i}(\varphi)+p_{i}(\varphi) \zeta(\varphi+\delta)\right), M_{j-}\right] d \varphi } \\
= & \lim _{\delta \rightarrow 0}\left(C_{i j}^{p \zeta, x \mathcal{H}}(\delta, 0)+C_{i j}^{p \zeta, p \zeta}(\delta, 0)\right) \\
= & \frac{2 \pi i}{\eta} \int\left\{-\left(\left(p_{i} x_{j} \mathcal{H}\right)\right)(\varphi)+\left(\left(x_{j} x_{i}^{\prime} \zeta^{\prime}\right)\right)(\varphi)-\frac{5}{2 \eta}\left(\left(x_{j}^{\prime \prime} p_{i}\right)\right)(\varphi)+\frac{3}{2 \eta}\left(\left(p_{j} x_{i}^{\prime \prime}\right)\right)(\varphi)+\frac{D-2}{6 \eta}\left(\left(x_{j}^{\prime \prime} p_{i}\right)\right)(\varphi)\right\} d \varphi
\end{aligned}
$$

Combining now all contributions we find for the quantum commutator the final result

$$
\left[M_{i-}, M_{j-}\right]=\frac{\pi i}{3 \eta^{2}}(D-26) \int\left(\left(\left(x_{j}^{\prime \prime} p_{i}\right)\right)(\varphi)-\left(\left(x_{i}^{\prime \prime} p_{j}\right)\right)(\varphi)\right) d \varphi
$$

which vanishes if $D=26$.

The dimension-dependent term (linear in $D-2$ ) came from the commutator of $\mathcal{H}$ and $\zeta$, the other anomalous terms came from all commutators that occur in the computation. As already remarked in the introduction, we did not introduce a normal-ordering constant to define $\mathcal{H}$. In principle such a shift $\left(\mathcal{H} \rightarrow \mathcal{H}+\frac{\text { const. }}{\eta}\right)$ could be considered (then one would derive from demanding a vanishing commutator $\left[M_{i-}, M_{j-}\right]$ that this constant is zero), but the definition of $\mathcal{H}$ via $\mathrm{OPE}$ normal ordering on the cylinder appears to be most natural.

\section{Acknowledgements}

We would like to thank Jürg Fröhlich and Volker Schomerus, AEI and KTH, and the Swedish Research Council.

\section{A Commutators of the form $\left[p_{i} \zeta, x_{j} \mathcal{H}\right]$}

We discuss here the contribution

$$
C_{i j}^{p \zeta, x \mathcal{H}}(\delta, \tilde{\epsilon})=-\frac{1}{2} \iint\left[\zeta(\varphi+\delta) p_{i}(\varphi)+p_{i}(\varphi) \zeta(\varphi+\delta), x_{j}(\tilde{\varphi}+\tilde{\epsilon}) \mathcal{H}(\tilde{\varphi})\right] d \varphi d \tilde{\varphi}
$$


For $i \neq j$ the commutator is given by

$$
\begin{aligned}
C_{i j}^{p \zeta, x \mathcal{H}}(\delta, \tilde{\epsilon})= & -\frac{2 \pi i}{\eta} \int\left(x_{j}(\varphi+\tilde{\epsilon}) p_{i}(\varphi-\delta) \mathcal{H}(\varphi)-x_{j}(\varphi+\tilde{\epsilon}-\delta) x_{i}^{\prime}(\varphi-\delta) \zeta^{\prime}(\varphi)\right) d \varphi \\
& -\frac{i}{\eta} \iint x_{j}^{\prime}(\tilde{\varphi}+\tilde{\epsilon}) p_{i}(\varphi) \mathcal{H}(\tilde{\varphi}) \partial_{\tilde{\varphi}}(G(\varphi+\delta, \tilde{\varphi}+\tilde{\epsilon})-G(\varphi+\delta, \tilde{\varphi})) d \varphi d \tilde{\varphi} \\
& +\frac{\pi i}{3 \eta^{2}}(D-2) \int x_{j}^{\prime \prime}(\varphi+\delta+\tilde{\epsilon}) p_{i}(\varphi) d \varphi
\end{aligned}
$$

The result is regular when we expand in $\tilde{\epsilon}$, and we obtain

$$
\begin{aligned}
C_{i j}^{p \zeta, x \mathcal{H}}(\delta, 0)=\frac{2 \pi i}{\eta} \int & \left\{-p_{i}(\varphi-\delta)\left(\left(x_{j} \mathcal{H}\right)\right)(\varphi)+\left(\left(x_{j} x_{i}^{\prime}\right)\right)(\varphi-\delta) \zeta^{\prime}(\varphi)\right. \\
& \left.-\frac{1}{2 \eta} x_{j}^{\prime \prime}(\varphi+\delta) p_{i}(\varphi)+\frac{D-2}{6 \eta} x_{j}^{\prime \prime}(\varphi+\delta) p_{i}(\varphi)\right\} d \varphi
\end{aligned}
$$

Expanding in $\delta$ we find

$$
\begin{aligned}
C_{i j}^{p \zeta, x \mathcal{H}}(\delta, 0)=\frac{2 \pi i}{\eta} \int & \left\{\frac{1}{\delta \eta}\left(\left(\left(p_{i} x_{j}^{\prime}\right)\right)(\varphi)-\left(\left(p_{j} x_{i}^{\prime}\right)\right)(\varphi)\right)-\left(\left(p_{i} x_{j} \mathcal{H}\right)\right)(\varphi)+\left(\left(\left(x_{j} x_{i}^{\prime}\right)\right) \zeta^{\prime}\right)\right)(\varphi) \\
& \left.-\frac{1}{2 \eta}\left(\left(x_{j}^{\prime \prime} p_{i}\right)\right)(\varphi)+\frac{D-2}{6 \eta}\left(\left(x_{j}^{\prime \prime} p_{i}\right)\right)(\varphi)\right\} d \varphi+\mathcal{O}(\delta) \\
=\frac{2 \pi i}{\eta} \int & \left\{\frac{1}{\delta \eta}\left(\left(\left(p_{i} x_{j}^{\prime}\right)\right)(\varphi)-\left(\left(p_{j} x_{i}^{\prime}\right)\right)(\varphi)\right)-\left(\left(p_{i} x_{j} \mathcal{H}\right)\right)(\varphi)+\left(\left(x_{j} x_{i}^{\prime} \zeta^{\prime}\right)\right)(\varphi)\right. \\
+ & \left.\frac{1}{\eta}\left(\left(x_{i}^{\prime \prime} p_{j}\right)\right)-\frac{1}{\eta}\left(\left(x_{j}^{\prime \prime} p_{i}\right)\right)(\varphi)+\frac{D-2}{6 \eta}\left(\left(x_{j}^{\prime \prime} p_{i}\right)\right)(\varphi)\right\} d \varphi+\mathcal{O}(\delta)
\end{aligned}
$$

\section{B Commutators of the form $\left[p_{i} \zeta, p_{j} \zeta\right]$}

Let us now discuss the term

$$
C_{i j}^{p \zeta, p \zeta}(\delta, \tilde{\delta})=\frac{1}{4} \iint\left[\zeta(\varphi+\delta) p_{i}(\varphi)+p_{i}(\varphi) \zeta(\varphi+\delta), \zeta(\tilde{\varphi}+\tilde{\delta}) p_{j}(\tilde{\varphi})+p_{j}(\tilde{\varphi}) \zeta(\tilde{\varphi}+\tilde{\delta})\right] d \varphi d \tilde{\varphi} .
$$

One can straightforwardly show that the four different terms that one obtains from expanding the commutator above all lead to the same contribution,

$$
\begin{aligned}
C_{i j}^{p \zeta, p \zeta}(\delta, \tilde{\delta}) & =\frac{1}{4} \iint\left[\left[\zeta(\varphi+\delta), p_{i}(\varphi)\right]+2 p_{i}(\varphi) \zeta(\varphi+\delta),\left[\zeta(\tilde{\varphi}+\tilde{\delta}), p_{j}(\tilde{\varphi})\right]+2 p_{j}(\tilde{\varphi}) \zeta(\tilde{\varphi}+\tilde{\delta})\right] d \varphi d \tilde{\varphi} \\
& =\iint\left[p_{i}(\varphi) \zeta(\varphi+\delta), p_{j}(\tilde{\varphi}) \zeta(\tilde{\varphi}+\tilde{\delta})\right] d \varphi d \tilde{\varphi}
\end{aligned}
$$


By explicitly evaluating the commutator we find

$$
\begin{aligned}
C_{i j}^{p \zeta, p \zeta}(\delta, \tilde{\delta})= & \frac{2 \pi i}{\eta} \int\left(p_{i}(\varphi-\delta-\tilde{\delta}) p_{j}(\varphi-\tilde{\delta})-p_{j}(\varphi-\delta-\tilde{\delta}) p_{i}(\varphi-\delta)\right) \zeta(\varphi) d \varphi \\
+ & \frac{i}{\eta} \iint\left\{p_{i}(\varphi-\delta) p_{j}^{\prime}(\tilde{\varphi}-\tilde{\delta}) \partial_{\tilde{\varphi}}(G(\varphi, \tilde{\varphi}-\tilde{\delta})-G(\varphi, \tilde{\varphi})) \zeta(\tilde{\varphi})\right. \\
& \left.-p_{j}(\tilde{\varphi}-\tilde{\delta}) p_{i}^{\prime}(\varphi-\delta) \partial_{\varphi}(G(\tilde{\varphi}, \varphi-\delta)-G(\tilde{\varphi}, \varphi)) \zeta(\varphi)\right\} d \varphi d \tilde{\varphi} \\
= & \frac{i}{\eta} \iint\left\{-p_{i}(\varphi-\delta) p_{j}(\tilde{\varphi}-\tilde{\delta}) \partial_{\tilde{\varphi}}(G(\varphi, \tilde{\varphi}-\tilde{\delta})-G(\varphi, \tilde{\varphi})) \zeta^{\prime}(\tilde{\varphi})\right. \\
& \left.+p_{j}(\tilde{\varphi}-\tilde{\delta}) p_{i}(\varphi-\delta) \partial_{\varphi}(G(\tilde{\varphi}, \varphi-\delta)-G(\tilde{\varphi}, \varphi)) \zeta^{\prime}(\varphi)\right\} d \varphi d \tilde{\varphi}
\end{aligned}
$$

We now expand this expression in $\tilde{\delta}$ analogously to the cases we discussed before. We obtain

$$
\begin{aligned}
C_{i j}^{p \zeta, p \zeta}(\delta, \tilde{\delta})= & \frac{i}{\eta} \iint\left\{-p_{i}(\varphi-\delta)\left(-\frac{1}{\eta \tilde{\delta}^{2}} x_{j}^{\prime}(\tilde{\varphi})+\left(\left(p_{j} \zeta^{\prime}\right)\right)(\tilde{\varphi})\right)\left(-\tilde{\delta} \partial_{\tilde{\varphi}}^{2} G(\varphi, \tilde{\varphi})+\frac{\tilde{\delta}^{2}}{2} \partial_{\tilde{\varphi}}^{3} G(\varphi, \tilde{\varphi})\right)\right. \\
& \quad+p_{i}(\varphi-\delta) \partial_{\varphi}(G(\tilde{\varphi}, \varphi-\delta)-G(\tilde{\varphi}, \varphi))\left(p_{j}(\tilde{\varphi}-\tilde{\delta}) \zeta^{\prime}(\varphi)-\frac{2 \pi}{\eta} S_{\operatorname{sing}}(\tilde{\varphi}-\tilde{\delta}, \varphi) x_{j}^{\prime}(\varphi)\right) \\
& \left.\quad+p_{i}(\varphi-\delta) \partial_{\varphi}(G(\tilde{\varphi}, \varphi-\delta)-G(\tilde{\varphi}, \varphi)) \frac{2 \pi}{\eta} S_{\operatorname{sing}}(\tilde{\varphi}-\tilde{\delta}, \varphi) x_{j}^{\prime}(\varphi)\right\} d \varphi d \tilde{\varphi}+\mathcal{O}(\tilde{\delta}) \\
= & \frac{\pi i}{\eta^{2}} \int\left(-\frac{2}{\tilde{\delta}} p_{i}(\varphi-\delta) x_{j}^{\prime}(\varphi)-p_{i}(\varphi-\delta) x_{j}^{\prime \prime}(\varphi)\right) d \varphi \\
& +\frac{i}{\eta} \iint p_{i}(\varphi-\delta) \partial_{\varphi}(G(\tilde{\varphi}, \varphi-\delta)-G(\tilde{\varphi}, \varphi))\left(p_{j}(\tilde{\varphi}) \zeta^{\prime}(\varphi)-\frac{2 \pi}{\eta} S_{\operatorname{sing}}(\tilde{\varphi}, \varphi) x_{j}^{\prime}(\varphi)\right) d \varphi d \tilde{\varphi} \\
& +\frac{\pi i}{\eta^{2}} \int p_{i}(\varphi-\delta) x_{j}^{\prime}(\varphi)\left(-\cos \frac{\delta+\tilde{\delta}}{2} \frac{1}{\sin \frac{\delta+\tilde{\delta}}{2}}+\cos \frac{\tilde{\delta}}{2} \frac{1}{\sin \frac{\tilde{\delta}}{2}}\right) d \varphi+\mathcal{O}(\tilde{\delta}) \\
= & \frac{\pi i}{\eta^{2}} \int\left(-p_{i}(\varphi-\delta) x_{j}^{\prime \prime}(\varphi)\right) d \varphi \\
& +\frac{i}{\eta} \iint p_{i}(\varphi-\delta) \partial_{\varphi}(G(\tilde{\varphi}, \varphi-\delta)-G(\tilde{\varphi}, \varphi))\left(p_{j}(\tilde{\varphi}) \zeta^{\prime}(\varphi)-\frac{2 \pi}{\eta} S_{\operatorname{sing}}(\tilde{\varphi}, \varphi) x_{j}^{\prime}(\varphi)\right) d \varphi d \tilde{\varphi} \\
& +\frac{\pi i}{\eta^{2}} \int p_{i}(\varphi-\delta) x_{j}^{\prime}(\varphi)\left(-\cos \frac{\delta+\tilde{\delta}}{2} \frac{1}{\sin \frac{\delta+\tilde{\delta}}{2}}\right) d \varphi+\mathcal{O}(\tilde{\delta})
\end{aligned}
$$


As expected there is no singularity in $\tilde{\delta}$. We now set $\tilde{\delta}=0$ and expand in $\delta$,

$$
\begin{aligned}
C_{i j}^{p \zeta, p \zeta}(\delta, 0)= & \frac{\pi i}{\eta^{2}} \int\left(-\left(\left(p_{i} x_{j}^{\prime \prime}\right)\right)(\varphi)\right) d \varphi \\
& +\frac{\pi i}{\eta^{2}} \int\left(\frac{2}{\delta}\left(\left(p_{j} x_{i}^{\prime}\right)\right)(\varphi)+\left(\left(p_{j} x_{i}^{\prime \prime}\right)\right)(\varphi)\right) d \varphi \\
& +\frac{\pi i}{\eta^{2}} \int\left(-\frac{2}{\delta}\left(\left(p_{i} x_{j}^{\prime}\right)\right)(\varphi)-2\left(\left(p_{i} x_{j}^{\prime \prime}\right)\right)(\varphi)\right) d \varphi+\mathcal{O}(\delta) \\
= & \frac{2 \pi i}{\eta} \int\left\{\frac{1}{\delta \eta}\left(\left(\left(p_{j} x_{i}^{\prime}\right)\right)(\varphi)-\left(\left(p_{i} x_{j}^{\prime}\right)\right)(\varphi)\right)\right. \\
& \left.+\left(-\frac{3}{2 \eta}\left(\left(p_{i} x_{j}^{\prime \prime}\right)\right)(\varphi)+\frac{1}{2 \eta}\left(\left(p_{j} x_{i}^{\prime \prime}\right)\right)(\varphi)\right)\right\} d \varphi+\mathcal{O}(\delta) .
\end{aligned}
$$

\section{Non-associativity}

OPE normal ordering is in general not associative. In this appendix we will discuss those cases that are relevant in the main text.

The first identity, we want to explain is

$$
\left.\left(\left(\left(x_{j} p_{i}\right)\right) \mathcal{H}\right)\right)=\left(\left(x_{j} p_{i} \mathcal{H}\right)\right)-\frac{1}{2 \eta}\left(\left(\left(x_{j}^{\prime \prime} p_{i}\right)\right)-2\left(\left(p_{i}^{\prime} x_{j}^{\prime}\right)\right)\right) .
$$

The simplest way to show this is to write

$$
\mathcal{H}=\sum_{k} \mathcal{H}_{k} \quad, \quad \mathcal{H}_{k}=\frac{\pi}{\eta}\left(\left(\left(p_{k}^{2}\right)\right)+\left(\left(x_{k}^{\prime 2}\right)\right)\right),
$$

and consider the summands $\mathcal{H}_{k}$ individually. For $k$ different from $i$ and $j$ there is no singularity, and thus normal ordering of $x_{j}, p_{i}$ and $\mathcal{H}_{k}$ is associative. Now consider $k=i$,

$$
\begin{aligned}
\left.\left(\left(\left(x_{j} p_{i}\right)\right) \mathcal{H}_{i}\right)\right)(\varphi) & =\lim _{\epsilon \rightarrow 0}\left(x_{j}(\varphi-\epsilon) p_{i}(\varphi-\epsilon) \mathcal{H}_{i}(\varphi)+\frac{1}{\epsilon^{2} \eta}\left(x_{j}(\varphi)-\epsilon x_{j}^{\prime}(\varphi)\right) p_{i}(\varphi)\right) \\
& =\lim _{\epsilon \rightarrow 0}\left(x_{j}(\varphi-\epsilon)\left(\left(\left(p_{i} \mathcal{H}_{i}\right)\right)(\varphi)-\frac{1}{\epsilon^{2} \eta} p_{i}(\varphi)\right)+\frac{1}{\epsilon^{2} \eta}\left(x_{j}(\varphi)-\epsilon x_{j}^{\prime}(\varphi)\right) p_{i}(\varphi)\right) \\
& =\left(\left(x_{j} p_{i} \mathcal{H}_{i}\right)\right)(\varphi)-\frac{1}{2 \eta}\left(\left(x_{j}^{\prime \prime} p_{i}\right)\right)(\varphi) .
\end{aligned}
$$

If we instead consider $k=j$, we find

$$
\begin{aligned}
\left.\left(\left(\left(x_{j} p_{i}\right)\right) \mathcal{H}_{j}\right)\right)(\varphi) & =\lim _{\epsilon \rightarrow 0}\left(x_{j}(\varphi-\epsilon) p_{i}(\varphi-\epsilon) \mathcal{H}_{j}(\varphi)+\frac{1}{\epsilon \eta} x_{j}^{\prime}(\varphi) p_{i}(\varphi)\right) \\
& =\lim _{\epsilon \rightarrow 0}\left(x_{j}(\varphi-\epsilon)\left(p_{i}(\varphi)-\epsilon p_{i}^{\prime}(\varphi)\right) \mathcal{H}_{j}(\varphi)+\frac{1}{\epsilon \eta} x_{j}^{\prime}(\varphi) p_{i}(\varphi)\right) \\
& =\lim _{\epsilon \rightarrow 0}\left(\left(\left(\left(x_{j} p_{i} \mathcal{H}_{j}\right)\right)(\varphi)-\frac{1}{\epsilon \eta} x_{j}^{\prime}(\varphi) p_{i}(\varphi)\right)+\frac{1}{\eta} x_{j}^{\prime}(\varphi) p_{i}^{\prime}(\varphi)+\frac{1}{\epsilon \eta} x_{j}^{\prime}(\varphi) p_{i}(\varphi)\right) \\
& =\left(\left(x_{j} p_{i} \mathcal{H}_{j}\right)\right)(\varphi)+\frac{1}{\eta}\left(\left(x_{j}^{\prime} p_{i}^{\prime}\right)\right)(\varphi) .
\end{aligned}
$$


Combining (C.5) and (C.9) we arrive at the desired result (C.1).

The second relation that we need is

$$
\left.\left(\left(\left(x_{i} x_{j}^{\prime}\right)\right) \zeta^{\prime}\right)\right)=\left(\left(x_{i} x_{j}^{\prime} \zeta^{\prime}\right)\right)+\frac{1}{2 \eta}\left(2\left(\left(x_{j}^{\prime \prime} p_{i}\right)\right)-\left(\left(x_{i}^{\prime \prime} p_{j}\right)\right)\right) .
$$

We can prove it analogously. Write

$$
\zeta^{\prime}=\sum_{k} \zeta_{k}^{\prime} \quad, \quad \zeta_{k}^{\prime}=\frac{2 \pi}{\eta}\left(\left(p_{k} x_{k}\right)\right)
$$

and consider first the case when $\zeta_{i}^{\prime}$ appears in the normal-ordered product,

$$
\begin{aligned}
\left.\left(\left(\left(x_{i} x_{j}^{\prime}\right)\right) \zeta_{i}^{\prime}\right)\right) & =\lim _{\epsilon \rightarrow 0}\left(x_{i}(\varphi-\epsilon) x_{j}^{\prime}(\varphi-\epsilon) \zeta_{i}^{\prime}(\varphi)+\frac{1}{\epsilon \eta} x_{j}^{\prime}(\varphi) p_{i}(\varphi)\right) \\
& =\lim _{\epsilon \rightarrow 0}\left(x_{i}(\varphi-\epsilon)\left(x_{j}^{\prime}(\varphi)-\epsilon x_{j}^{\prime \prime}(\varphi)\right) \zeta_{i}^{\prime}(\varphi)+\frac{1}{\epsilon \eta} x_{j}^{\prime}(\varphi) p_{i}(\varphi)\right) \\
& =\lim _{\epsilon \rightarrow 0}\left(\left(\left(\left(x_{i} x_{j}^{\prime} \zeta_{i}^{\prime}\right)\right)(\varphi)-\frac{1}{\epsilon \eta} x_{j}^{\prime}(\varphi) p_{i}(\varphi)\right)+\frac{1}{\eta} x_{j}^{\prime \prime}(\varphi) p_{i}(\varphi)+\frac{1}{\epsilon \eta} x_{j}^{\prime}(\varphi) p_{i}(\varphi)\right) \\
& =\left(\left(x_{i} x_{j}^{\prime} \zeta_{i}^{\prime}\right)\right)(\varphi)+\frac{1}{\eta}\left(\left(x_{j}^{\prime \prime} p_{i}\right)\right)(\varphi) .
\end{aligned}
$$

Now consider the case, when $\zeta_{j}^{\prime}$ occurs,

$$
\begin{aligned}
\left.\left(\left(\left(x_{i} x_{j}^{\prime}\right)\right) \zeta_{j}^{\prime}\right)\right) & =\lim _{\epsilon \rightarrow 0}\left(x_{i}(\varphi-\epsilon) x_{j}^{\prime}(\varphi-\epsilon) \zeta_{j}^{\prime}(\varphi)+\frac{1}{\epsilon^{2} \eta}\left(x_{i}(\varphi)-\epsilon x_{i}^{\prime}(\varphi)\right) p_{j}(\varphi)\right) \\
& =\lim _{\epsilon \rightarrow 0}\left(x_{i}(\varphi-\epsilon)\left(\left(\left(x_{j}^{\prime} \zeta_{j}^{\prime}\right)\right)(\varphi)-\frac{1}{\epsilon^{2} \eta} p_{j}(\varphi)\right)+\frac{1}{\epsilon^{2} \eta}\left(x_{i}(\varphi)-\epsilon x_{i}^{\prime}(\varphi)\right) p_{j}(\varphi)\right) \\
& =\left(\left(x_{i} x_{j}^{\prime} \zeta_{j}^{\prime}\right)\right)(\varphi)-\frac{1}{2 \eta}\left(\left(x_{i}^{\prime \prime} p_{j}\right)\right)(\varphi) .
\end{aligned}
$$

Combining (C.15) and (C.18) we arrive at the final result (C.10).

\section{References}

[1] C. Lovelace, Pomeron form-factors and dual Regge cuts, Phys. Lett. B34 (1971) 500.

[2] R.C. Brower, Spectrum generating algebra and no ghost theorem for the dual model, Phys. Rev. D 6 (1972) 1655.

[3] P. Goddard and C.B. Thorn, Compatibility of the Dual Pomeron with Unitarity and the Absence of Ghosts in the Dual Resonance Model, Phys. Lett. B40 (1972) 235.

[4] P. Goddard, J. Goldstone, C. Rebbi and C.B. Thorn, Quantum dynamics of a massless relativistic string, Nucl. Phys. B56 (1973) 109.

[5] G. Arutyunov, Lectures on String Theory, Utrecht University 2009

[6] J. Hoppe, Fundamental Structures of M(brane) Theory, Phys. Lett. B 695 (2011) 384, arXiv:1003.5189 [hep-th]]. 
[7] U. Marquard and M. Scholl, Lorentz Algebra and Critical Dimension for the Bosonic Membrane, Phys. Lett. B227 (1989) 227.

[8] K. Bering, A Note on Angular Momentum Commutators in Light-Cone Formulation of Open Bosonic String Theory, Acta Phys. Polon. B43 (2012) 1811 arXiv:1104.4446 [hep-th]].

[9] J. Hoppe, M-brane dynamical symmetry and quantization, arXiv:1101.4334 [hep-th].

[10] J. Goldstone, unpublished notes (1985) 